\title{
LOCAL SYSTEMS ON COMPLEMENTS OF ARRANGEMENTS OF SMOOTH, COMPLEX ALGEBRAIC HYPERSURFACES
}

\author{
GRAHAM DENHAM ${ }^{1}$ and ALEXANDER I. SUCIU ${ }^{2}$ \\ ${ }^{1}$ Department of Mathematics, University of Western Ontario, London, ON N6A 5B7, Canada; \\ email: gdenham@uwo.ca \\ 2 Department of Mathematics, Northeastern University, Boston, MA 02115, USA; \\ email: a.suciu@northeastern.edu
}

Received 19 June 2017; accepted 24 March 2018

\begin{abstract}
We consider smooth, complex quasiprojective varieties $U$ that admit a compactification with a boundary, which is an arrangement of smooth algebraic hypersurfaces. If the hypersurfaces intersect locally like hyperplanes, and the relative interiors of the hypersurfaces are Stein manifolds, we prove that the cohomology of certain local systems on $U$ vanishes. As an application, we show that complements of linear, toric, and elliptic arrangements are both duality and abelian duality spaces.
\end{abstract}

2010 Mathematics Subject Classification: 55N25 (primary); 14M27, 20J05, 32E10, 32S22, 55P62, 55R80, 55U30, 57M07 (secondary)

\section{Introduction}

1.1. Abelian duality and local systems. It has long been recognized that complements of complex hyperplane arrangements satisfy certain vanishing properties for homology with coefficients in local systems. We revisited this subject in our joint work with Yuzvinsky, $[13,14]$, in a more general context.

Let $X$ be a connected, finite-type CW-complex, with a fundamental group $G$. Following Bieri and Eckmann [3], we say that $X$ is a duality space of dimension $n$ if $H^{q}(X, \mathbb{Z} G)=0$ for $q \neq n$ and $H^{n}(X, \mathbb{Z} G)$ is nonzero and torsion-free. We also say that $X$ is an abelian duality space of dimension $n$ if the analogous condition, with the coefficient $G$-module $\mathbb{Z} G$ replaced by $\mathbb{Z} G^{\text {ab }}$ is satisfied. 
Setting $D:=H^{n}\left(X, \mathbb{Z} G^{\mathrm{ab}}\right)$, it follows that $H^{i}(X, A) \cong H_{n-i}\left(G^{\mathrm{ab}}, D \otimes_{\mathbb{Z}} A\right)$ for any representation $A$ of $G$, which factors through $G^{\mathrm{ab}}$, and for all $i \geqslant 0$.

It is worth noting that the abelian duality property imposes significant conditions on the cohomology of local systems on $X$. Let $\mathbb{k}$ be an algebraically closed field. The group $\widehat{G}=\operatorname{Hom}_{\mathrm{Gps}}\left(G, \mathbb{k}^{*}\right)$ of $\mathbb{k}$-valued multiplicative characters of $G$ is an algebraic group, with identity the trivial representation 1 . The characteristic varieties $\mathcal{V}^{q}(X, \mathbb{k})$ are the subsets of $\widehat{G}$ consisting of those characters $\rho$ for which $H^{q}\left(X, \mathbb{k}_{\rho}\right) \neq 0$. We highlight an interesting consequence of the abelian duality space property, which was established in [14]: if $X$ is an abelian duality space of dimension $n$, then the characteristic varieties of $X$ propagate, that is,

$$
\{\mathbf{1}\}=\mathcal{V}^{0}(X, \mathbb{k}) \subseteq \mathcal{V}^{1}(X, \mathbb{k}) \subseteq \cdots \subseteq \mathcal{V}^{n}(X, \mathbb{k}),
$$

or, equivalently, if $H^{p}\left(X, \mathbb{k}_{\rho}\right) \neq 0$ for some $\rho \in \widehat{G}$, then $H^{q}\left(X, \mathbb{k}_{\rho}\right) \neq 0$ for all $p \leqslant q \leqslant n$.

The abelian duality property also imposes stringent conditions on the cohomology groups $H^{i}(X, \mathbb{Z})=\operatorname{Tor}_{n-i}(D, \mathbb{Z})$. For instance, all the Betti numbers $b_{i}(X)$ must be positive for $0 \leqslant i \leqslant n$ and vanish for $i>n$, while $b_{1}(X) \geqslant n$. Furthermore, as noted in [33, Theorem 1.8], the results of [14] imply the following inequality for the 'signed Euler characteristic' of an abelian duality space of dimension $n$ :

$$
(-1)^{n} \chi(X) \geqslant 0 .
$$

1.2. Arrangements of smooth hypersurfaces. Davis et al. showed in [9] that complements of (linear) hyperplane arrangements are duality spaces. More generally, we proved in [14] that complements of both linear and elliptic arrangements are duality and abelian duality spaces.

Our goal here is to further generalize these results to a much wider class of arrangements of hypersurfaces, by which we mean a collection of smooth, irreducible, codimension 1 subvarieties which are embedded in a smooth, connected, complex projective algebraic variety, and which intersect locally like hyperplanes. We isolate a subclass of such arrangements whose complements enjoy the aforementioned duality properties, and therefore have vanishing twisted cohomology in the appropriate range.

THEOREM 1.1. Let $U$ be a connected, smooth, complex quasiprojective variety of dimension $n$. Suppose $U$ has a smooth compactification $Y$ for which:

(1) Components of the boundary $D=Y \backslash U$ form a nonempty arrangement of hypersurfaces $\mathcal{A}$. 
(2) For each submanifold $X$ in the intersection poset $L(\mathcal{A})$, the complement of the restriction of $\mathcal{A}$ to $X$ is either empty or a Stein manifold.

Then $U$ is both a duality space and an abelian duality space of dimension $n$.

An important consequence of this theorem is that the characteristic varieties of such 'recursively Stein' hypersurface complements propagate. As another application of Theorem 1.1, we prove in Corollary 2.9 the following 'generic vanishing of cohomology' result. We use here De Concini and Procesi's [12] construction of wonderful models for subspace arrangements, based on the notion of 'building sets'; see [21] for an exposition.

THEOREM 1.2. Let $U$ be a smooth, quasiprojective variety of dimension $n$ satisfying conditions (1) and (2) from above. Furthermore, let $G=\pi_{1}(U)$, and let $A$ be a finite-dimensional representation of $G$ over a field $\mathbb{k}$. Suppose that $A^{\gamma_{s}}=0$ for all $g$ in a building set $\mathcal{G}_{X}$, where $X \in L(\mathcal{A})$; then $H^{i}(U, A)=0$ for all $i \neq n$.

Consequently, the cohomology groups of $U$ with coefficients in a 'generic' local system vanish in the range below $n$.

Finally, let $\ell_{2} G$ denote the left $\mathbb{C}[G]$-module of complex-valued, squaresummable functions on $G$, and let ${ }^{\text {red }} H^{i}\left(U, \ell_{2} G\right)$ be the reduced $L^{2}$-cohomology groups of $U$ with coefficients in this module, cf. [17, 34]. We then prove in Theorem 2.11 the following result.

THEOREM 1.3. Let $U$ and $G=\pi_{1}(U)$ be as above. Then ${ }^{\mathrm{red}} H^{i}\left(U, \ell_{2} G\right)=0$ for all $i \neq n$.

In particular, the $\ell_{2}$-Betti numbers of $U$ are all zero except in dimension $n$. A basic fact about $\ell_{2}$-cohomology is that $\ell_{2}$-Betti numbers compute the usual Euler characteristic (see, e.g., [17, Theorem 3.6.1]). Therefore, we see once again that $(-1)^{n} \chi(U) \geqslant 0$.

1.3. Linear, elliptic, and toric arrangements. The theory of hyperplane arrangements originates in the study of configuration spaces and braid groups. Here we consider a broader class of hypersurface arrangements of current interest.

THEOREM 1.4. Suppose that $\mathcal{A}$ is one of the following:

(1) an affine-linear arrangement in $\mathbb{C}^{n}$, or a hyperplane arrangement in $\mathbb{C} \mathbb{P}^{n}$;

(2) a nonempty elliptic arrangement in $E^{n}$;

(3) a toric arrangement in $\left(\mathbb{C}^{*}\right)^{n}$. 
Then the complement $M(\mathcal{A})$ is both a duality space and an abelian duality space of dimension $n-r, n+r$, and $n$, respectively, where $r$ is the corank of the arrangement.

As mentioned previously, the first two statements already appeared in our paper [14]; at the time, however, we were unable to address the third one. Since then, De Concini and Gaiffi [11] have constructed a compactification for toric arrangements, which is compatible with our approach. The claim that the complement of a toric arrangement is a duality space was first reported in [10, Theorem 5.2]. However, a serious gap appeared in the proof; see [8]. Part of our motivation here, then, is to provide an independent alternative, as well as a uniform proof of the three claims above. The argument is given in Section 3.1 and depends on Theorem 1.1. It is worth noting that this dependency is slightly subtle. For example, the complement $U$ of a linear hyperplane arrangement is indeed a Stein manifold; however, if the corank is strictly positive, hypothesis (2) is not satisfied by the intersection of all the hyperplanes.

As a consequence of Theorem 1.4, the characteristic varieties propagate for all linear, elliptic, and toric arrangements. The formality of linear and toric arrangement complements implies that their resonance varieties propagate, as well. In the linear case, a more refined propagation of resonance property was established by Budur in [4].

If $\mathcal{A}$ is an affine complex arrangement, the work of Kohno [29], Esnault et al. [18], and Schechtman et al. [36] gives sufficient conditions for a local system $\mathcal{L}$ on $M(\mathcal{A})$ to ensure the vanishing of the cohomology groups $H^{i}(M(\mathcal{A}), \mathcal{L})$ for all $i<\operatorname{rank}(\mathcal{A})$. Similar conditions for the vanishing of cohomology with coefficients in rank 1 local systems were given by Levin and Varchenko [30] for elliptic arrangements, and by Esterov and Takeuchi [19] for certain toric hypersurface arrangements. In turn, we provide in Corollary 2.9 a unified set of generic vanishing conditions for cohomology of local systems on complements of arrangements of smooth, complex algebraic hypersurfaces.

The $\ell_{2}$-cohomology of the complement of a linear arrangement also vanishes outside of the middle (real) dimension: this is a result of Davis et al. [7]. The same claim for toric arrangements appears in [10]; however, the argument given there has the same gap mentioned above. As part of our approach here, we obtain the aforementioned vanishing result (Theorem 2.11) for the $\ell_{2}$-cohomology of complements of hypersurface arrangements.

1.4. Orbit configuration spaces. As a second application of our general results, we obtain an almost complete characterization of the duality and abelian duality properties of ordered orbit configuration spaces on Riemann surfaces. In 
Section 3.2, we define and discuss orbit configuration spaces, following [39]; for the purpose of this introduction, though, we remind the reader that the classical configuration spaces are recovered by taking $\Gamma$ to be the trivial group.

THEOREM 1.5. Suppose $\Gamma$ is a finite group that acts freely on a Riemann surface $\Sigma_{g, k}$ of genus $g$ with $k$ punctures. Let $F_{\Gamma}\left(\Sigma_{g, k}, n\right)$ be the orbit configuration space of $n$-ordered, disjoint $\Gamma$-orbits.

(1) If $k>0$, then $F_{\Gamma}\left(\Sigma_{g, k}, n\right)$ is both a duality space and an abelian duality space of dimension $n$.

(2) If $k=0$, then $F_{\Gamma}\left(\Sigma_{g}, n\right)$ is a duality space of dimension $n+1$, provided $g \geqslant 1$, and is an abelian duality space of dimension $n+1$ if $g=1$.

(3) If $k=0$, then $F\left(\Sigma_{g}, n\right)$ is neither a duality space nor an abelian duality space if $g=0$, and it is not an abelian duality space if $g \geqslant 2$.

Hence the characteristic varieties propagate for the orbit configuration spaces $F_{\Gamma}\left(\Sigma_{g, k}, n\right)$, where either $k \geqslant 1$, or $k=0$ and $g=1$, for any finite group $\Gamma$ acting freely on $\Sigma_{g, k}$.

\section{Hypersurface arrangements}

2.1. Open covers for hypersurface arrangements. Let $Y$ be a smooth, connected complex manifold, and let $\mathcal{A}=\left\{W_{1}, \ldots, W_{m}\right\}$ be a finite collection of smooth, connected, codimension-1 submanifolds of $Y$. Let $D=\bigcup_{i=1}^{m} W_{i}$ be the corresponding divisor, and let $M(\mathcal{A}):=Y \backslash D$ be the complement of the arrangement $\mathcal{A}$.

We will assume that the intersection of any subset of $\mathcal{A}$ is also a smooth manifold, and has only finitely many connected components. Moreover, we require that, for each point $y \in D$, there is a chart containing $y$ for which each element of the subcollection $\mathcal{A}_{y}:=\left\{W_{i} \mid y \in W_{i}\right\}$ is defined locally by a linear equation. In other words, the hypersurfaces comprising $\mathcal{A}$ have intersections which, locally, are diffeomorphic to hyperplane arrangements. This definition is taken from Dupont [16], though similar notions appear in the literature; in particular, we refer to the paper of $\mathrm{Li}$ [31].

Let $L(\mathcal{A})$ denote the collection of all connected components of intersections of zero or more of the hypersurfaces comprising $\mathcal{A}$. Then $L(\mathcal{A})$ forms a finite poset under reverse inclusion, ranked by codimension. We will write $X \leqslant Y$ if $X \supseteq Y$, and write $r(X)=\operatorname{codim} X$ for the rank function. For every submanifold $X \in L(\mathcal{A})$, let $\mathcal{A}_{X}=\{W \in \mathcal{A} \mid X \subseteq W\}$ be the closed subarrangement associated 
to $X$, and let $\mathcal{A}^{X}=\left\{W \cap X \mid W \in \mathcal{A} \backslash \mathcal{A}_{X}\right\}$ be the restriction of $\mathcal{A}$ to $X$. We write

$$
D_{X}=\bigcup_{Z \in L(\mathcal{A}): Z<X} Z
$$

Then the complement of the restriction of $\mathcal{A}$ to $X$ is $M\left(\mathcal{A}^{X}\right):=X \backslash D_{X}$, for each $X \in L(\mathcal{A})$. Finally, let $T \mathcal{A}_{X}$ be the hyperplane arrangement in the tangent space to $Y$ at a point in the relative interior of $X$, guaranteed by our hypothesis on the intersection of hypersurfaces.

One of the main tools we will need in this note is a spectral sequence developed in [13], which we summarize in the next theorem.

THEOREM 2.1 [13]. Let $\mathcal{A}$ be an arrangement of hypersurfaces in a compact, smooth manifold $Y$. Let $M$ be the complement of the arrangement, and let $\mathcal{F}$ be a locally constant sheaf on $M$. There is then a spectral sequence with

$$
E_{2}^{p q}=\prod_{X \in L(\mathcal{A})} H_{c}^{p+r(X)}\left(M\left(\mathcal{A}^{X}\right) ; H^{q-r(X)}\left(M\left(T \mathcal{A}_{X}\right), \mathcal{F}_{X}\right)\right),
$$

converging to $H^{p+q}(M, \mathcal{F})$, where $\mathcal{F}_{X}$ is the corresponding restriction of $\mathcal{F}$ to $M\left(T \mathcal{A}_{X}\right)$.

REMARK 2.2. This is a special case of [13, Corollary 3.3]. The indexing differs slightly since [13] indexes by dimension, rather than codimension.

2.2. Wonderful compactifications. From here on, we will consider arrangements $\mathcal{A}$ of smooth, algebraic hypersurfaces in a smooth, connected complex projective variety $Y$. For each $x \in Y$, there is a linear hyperplane arrangement $T \mathcal{A}_{X}$ in the complex vector space $V=T_{x} Y$ tangent to $\mathcal{A}_{X}$, where

$$
X=\bigcap_{x \in Z \in L(\mathcal{A})} Z \text {. }
$$

We apply De Concini and Procesi's construction of the wonderful model of a subspace arrangement [12] to the linear arrangement $T \mathcal{A}_{X}$ inside the affine space $V$. The construction blows up the arrangement to one with simple normal crossings; let $p: \widetilde{V} \rightarrow V$ denote the blowup. The (total) divisor components are indexed by a 'building set' $\mathcal{G}_{X}$. A subset $S \subseteq \mathcal{G}_{X}$ indexing divisor components that have nonempty intersection is called a 'nested set,' and the collection of all nested sets forms a simplicial complex, called the nested set complex. The nested set complex $\mathscr{N}\left(T \mathcal{A}_{X}\right)$ depends on the choice of building set; however, we will assume a fixed choice is made for each $X$ and omit the building set from the notation, since the choices are not important to what follows. 
2.3. An injectivity result. We recall from [13] that the fundamental group of the complement of a linear arrangement contains certain distinguished free abelian subgroups of finite rank. We review the definition of these subgroups briefly, referring to $[13,21]$ for unexplained terminology.

We consider the wonderful model for $T \mathcal{A}_{X}$ in the tangent space $V=V_{x}$ for a point $x$ in the relative interior of $X$. For a nested set $S \in \mathscr{N}\left(T \mathcal{A}_{X}\right)$ of size $r$, let $D_{S}$ denote the corresponding intersection of $r$ divisor components in $\widetilde{V}$. For a point $z$ in the relative interior of $D_{S}$, let $\mathbb{D}_{z}$ be a sufficiently small closed polydisc in $\widetilde{V}$ centered at $z$, as shown in Figure 1 . Then $U_{S}:=\mathbb{D}_{z} \cap M\left(T \mathcal{A}_{X}\right) \simeq\left(S^{1}\right)^{r}$. It is shown in [13, Theorem 4.6] that the inclusion $f_{X, S}: U_{S} \hookrightarrow M\left(T \mathcal{A}_{X}\right)$ induces an injective map of fundamental groups, $\left(f_{X, S}\right)_{\sharp}: \pi_{1}\left(U_{S}, z_{X, S}\right) \hookrightarrow \pi_{1}\left(M\left(T \mathcal{A}_{X}\right)\right.$, $\left.z_{X, S}\right)$. Let $C_{S}$ denote the image of this homomorphism. This is a free abelian subgroup of $G_{X}:=\pi_{1}\left(M\left(T \mathcal{A}_{X}\right)\right)$ of rank $r$, well defined up to conjugacy.

Our goal now is to show that $C_{S}$ also injects in the fundamental group of the hypersurface complement. Let $\mathbb{D}$ be a closed polydisc in $V=V_{x}$ centered at the origin, and set $V_{X}:=\mathbb{D} \backslash \bigcup_{W \in \mathcal{H}_{X}} T_{x} W \cap \mathbb{D}$. Let $h$ denote the diffeomorphism that trivializes $\left.T Y\right|_{\mathbb{D}}$, and let $\mathbb{D}_{x}$ be the image of $\mathbb{D}$ under $h$. Let $U_{X}=h\left(V_{X}\right)$; then $U_{X}=\mathbb{D}_{x} \backslash \bigcup_{W \in \mathcal{A}_{X}} W \cap \mathbb{D}_{x}$.

Consider the diagram

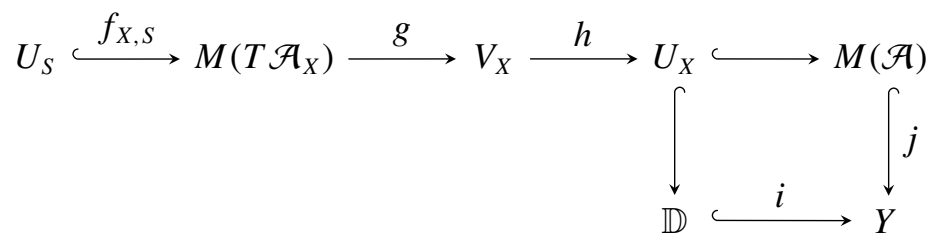

Here, $g$ is the usual deformation retraction of a central, linear arrangement complement, and the composite of the maps on the top row, $U_{S} \rightarrow M(\mathcal{A})$, induces a homomorphism $\alpha_{X, S}: \pi_{1}\left(U_{S}\right) \rightarrow \pi_{1}(M(\mathcal{A}))$.

Our argument for the injectivity of this map uses some rational homotopy theory. We start with a technical lemma. A connected CDGA $(A, d)$ is said to be 1-minimal if $A=\bigwedge V$, the free CDGA on a vector space $V$ concentrated in degree 1 , and $V$ is the union of an increasing filtration by subspaces $\left\{V_{i}\right\}_{i \geqslant 0}$ such that $d=0$ on $V_{0}$ and $d\left(V_{i+1}\right) \subset \bigwedge V_{i}$. (The differential $d$ is then decomposable, and thus $A$ is a minimal Sullivan algebra in the sense of [24].)

LEMMA 2.3. Let $A$ be a 1-minimal CDGA, and let $\varphi, \psi: A \rightarrow B$ be two homotopic CDGA morphisms. Suppose $d_{B}=0$ and $\varphi^{1}: A^{1} \rightarrow B^{1}$ is surjective. Then $\psi^{1}: A^{1} \rightarrow B^{1}$ is also surjective. 


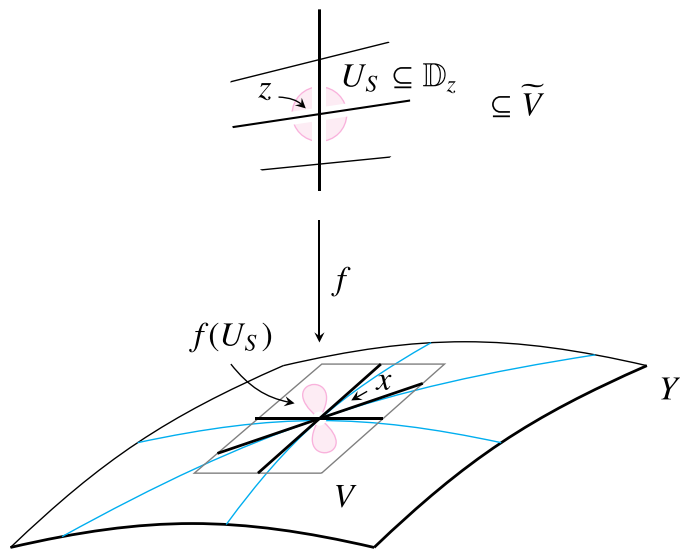

Figure 1. Small tori in $M(\mathcal{A})$.

Proof. Suppose $\psi^{1}$ is not surjective. Let us view $C:=\mathbb{k} \oplus B^{1} / \operatorname{im}\left(\psi^{1}\right)$ as a (connected) CDGA with zero differential, and let pr: $B \rightarrow C$ be the projection map, which sends the graded ideal generated by $\operatorname{im}\left(\psi^{1}\right)$ to zero. Clearly, the CDGA maps $\Phi=\operatorname{pr} \circ \varphi$ and $\Psi=\operatorname{pr} \circ \psi$ are homotopic, and $\Psi=0$; thus, $\Phi$ is null-homotopic. By [24, Example 1, p. 151], the map $\Phi$ is constant: that is, zero in positive degree. This implies that $\varphi^{1}$ is not surjective, a contradiction.

The complement of an arrangement of hypersurfaces $\mathcal{A}$ in a smooth, projective variety $Y$ has a rational CDGA model, given by the $E_{2}$-page of the Leray spectral sequence of the inclusion $j: M(\mathcal{A}) \rightarrow Y$. In the normal crossing case, this is the classical Morgan model; for configuration spaces it was used by Totaro [38]; in our more general context, we refer to Dupont [16] and Bibby [2]. We will denote this model for $M(\mathcal{A})$ by $B(\mathcal{A})$.

LEMMA 2.4. Let $\mathcal{A}$ be an arrangement of smooth, complex subvarieties in a smooth, complex projective variety $Y$. Then, for every $X \in L(\mathcal{A})$ and every nested set $S \in \mathscr{N}\left(T \mathcal{A}_{X}\right)$, the homomorphism $\alpha_{X, S}: \pi_{1}\left(U_{S}\right) \rightarrow \pi_{1}(M(\mathcal{A}))$ is injective.

Proof. Restriction along the map $i: \mathbb{D} \rightarrow Y$ gives a map of sheaves $\mathbb{Q} \rightarrow i_{*} \mathbb{Q}$ on $M(\mathcal{A})$, which in turn gives a map between the $E_{2}$-pages of the respective Leray spectral sequences,

$$
i^{*}: H^{p}\left(Y, R^{q} j_{*} \mathbb{Q}\right) \longrightarrow H^{p}\left(\mathbb{D}, R^{q} j_{*} \mathbb{Q}\right) .
$$

We note that, for each $q \geqslant 0$, the sheaf $R^{q} j_{*} \mathbb{Q}$ is the direct image of a constant sheaf on a contractible space, cf. [2, Lemma 3.1]; it follows that 
$H^{p}\left(\mathbb{D}, R^{q} j_{*} \mathbb{Q}\right)=H^{q}\left(U_{X}, \mathbb{Q}\right)$ for $p=0$, and is zero otherwise. Thus, the $E_{2}$-page on the right side of (7) is a CDGA with zero differential, isomorphic to the Orlik-Solomon algebra $\operatorname{OS}_{X}:=H^{\bullet}\left(M\left(T \mathcal{A}_{X}\right), \mathbb{Q}\right)$, and a rational model for $U_{X}$.

Now recall from [16] that the $\operatorname{CDGA} B(\mathcal{A})$ contains distinguished elements in bidegree $(0,1)$, which we denote by $\left\{e_{W} \mid W \in \mathcal{A}\right\}$, that restrict locally to logarithmic 1-forms around the components of $\mathcal{A}$ in $Y$. Since their images $i^{*}\left(e_{W}\right)$ for $W \in \mathcal{A}_{X}$ are the standard generators of $\mathrm{OS}_{X}$, the map $i^{*}$ is surjective. Recall also that we identified the group $\pi_{1}\left(U_{S}\right)$ with $\mathbb{Z}^{r}$, for some $r>0$. By [22, Proposition 2], or the proof of [13, Corollary 4.7], the map $f_{X, S}^{*}: \mathrm{OS}_{X} \rightarrow$ $H^{\bullet}\left(\left(\mathbb{C}^{*}\right)^{r}, \mathbb{Q}\right)$ is surjective in degree 1 . Hence, the composite $p=f_{X, S}^{*} \circ$ $i^{*}: B(\mathcal{A}) \rightarrow \bigwedge\left(\mathbb{Q}^{r}\right)$ is a surjective map of CDGAs, where the differential in $\bigwedge\left(\mathbb{Q}^{r}\right)$ is zero.

As is well known, every CDGA $A$ has a 1-minimal model, $\mathrm{M}(A)$, which is unique up to homotopy; see e.g. [28]. Such a model comes equipped with a morphism $\pi: \mathrm{M}(A) \rightarrow A$ inducing an isomorphism on $H^{1}$ and a monomorphism on $H^{2}$. We thus obtain a commuting square,

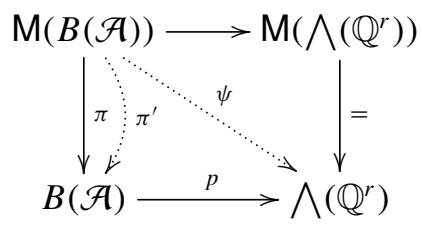

noting that the rational exterior algebra is its own minimal model. Let $\varphi=p \circ \pi$. Since both $H^{1}(p)$ and $H^{1}(\pi)$ are surjective, the map $H^{1}(\varphi)$ is also surjective. Since the differential of $\bigwedge\left(\mathbb{Q}^{r}\right)$ is zero, the map $\varphi$ itself is surjective in degree 1 .

Let $\mathfrak{m}(G)$ be the pronilpotent Lie algebra associated to $G=\pi_{1}(M(\mathcal{A}), x)$. As it is again well known, $\mathfrak{m}(G)$ coincides with the Lie algebra dual to the 1minimal model $\mathrm{M}(B(\mathcal{A}))$; we refer to [28] and also [37, Section 7] for discussion and further references. Let $\psi: \mathrm{M}(B(\mathcal{A})) \rightarrow \bigwedge\left(\mathbb{Q}^{r}\right)$ be the CDGA morphism dual to the Lie algebra map $\mathfrak{m}\left(\alpha_{X, S}\right): \mathfrak{m}\left(\mathbb{Z}^{r}\right) \rightarrow \mathfrak{m}(G)$. By minimality of $\mathrm{M}(B(\mathcal{A}))$, the map $\psi$ lifts to a map $\pi^{\prime}: \mathrm{M}(B(\mathcal{A})) \rightarrow B(\mathcal{A})$; see [24, Lemma 12.4]. By construction, $\pi^{\prime}$ is a classifying map for the 1-minimal model of $B(\mathcal{A})$; thus, $\pi \simeq \pi^{\prime}$, and so $\varphi \simeq \psi$. By Lemma 2.3, the map $\psi$ is surjective in degree 1; hence, $\mathfrak{m}\left(\alpha_{X, S}\right)$ is injective.

Let $\left(\alpha_{X, S}\right)_{\mathbb{Q}}:\left(\mathbb{Z}^{r}\right)_{\mathbb{Q}} \rightarrow G_{\mathbb{Q}}$ be the induced homomorphism between rational, prounipotent completions. By the above, this homomorphism is also injective. Since $\mathbb{Z}^{r}$ is a finitely generated, torsion-free abelian group, the natural map $\iota=$ $\mathbb{Z}^{r} \rightarrow\left(\mathbb{Z}^{r}\right)_{\mathbb{Q}}$ is injective, as well. Hence, the map $\alpha_{X, S}=\left(\alpha_{X, S}\right)_{\mathbb{Q}} \circ \iota$ itself is injective, and we are done. 
2.4. The main result. The cohomological vanishing results in [13] made use of the following condition on $G$-modules.

DEFINITION 2.5. Let $\mathbb{k}=\mathbb{Z}$ or a field, let $R=\mathbb{k}\left[\mathbb{Z}^{n}\right]$ for some $n \geqslant 1$, and let $I_{1}$ be the augmentation ideal of $R$. We say that an $R$-module $A$ is a maximal Cohen-Macaulay (MCM) module provided that $\operatorname{depth}_{R}\left(I_{1}, A\right) \geqslant n$. We note that this is slightly weaker than the usual notion, since we do not assume $A$ is finitely generated, and we allow the possibility that $\operatorname{Ext}_{R}\left(R / I_{1}, A\right)$ is identically zero, in which case we take $\operatorname{depth}_{R}\left(I_{1}, A\right)=\infty$.

Now let $\mathcal{A}$ be an arrangement of smooth, complex subvarieties in a smooth, complex variety $Y$, and let $G=\pi_{1}(M(\mathcal{A})$ ). From now on, we will assume $Y$ is compact. In view of Lemma 2.4, we will let $C_{S, X}$ denote the conjugacy class of the subgroup $\alpha_{X, x}\left(C_{S}\right)<G$, a free abelian group of rank $|S|$. We then extend the previous definition to this context, as follows.

Definition 2.6. Let $A$ be a (left) $\mathbb{k}[G]$-module. We say that $A$ is an MCM module provided that the restriction of $A$ to each subalgebra $\mathbb{k}\left[C_{S, X}\right]$ is MCM, for all flats $X \in L(\mathcal{A})$ and all nested sets $S \in \mathscr{N}\left(T \mathcal{A}_{X}\right)$.

Before proceeding, we need to recall some well-known facts about Stein manifolds; see e.g. [26, 27]. A complex manifold $M$ is said to be a Stein manifold if it can be realized as a closed, complex submanifold of some complex affine space. Alternatively, holomorphic functions on $M$ separate points, and $M$ is holomorphically convex. The Stein property is preserved under taking closed submanifolds and finite direct products. Furthermore, every Stein manifold of (complex) dimension $n$ has the homotopy type of a CW-complex of dimension $n$.

We are now in a position to state and prove our main vanishing-of-cohomology result.

THEOREM 2.7. Let $\mathcal{A}$ be an arrangement of hypersurfaces in a compact, complex, smooth variety $Y$ of dimension $n$. Suppose that $M\left(\mathcal{A}^{X}\right)$ is Stein for each $X \in L(\mathcal{A})$. Then, for any $\mathrm{MCM}$ module $A$ on $M(\mathcal{A})$, we have $H^{p}(M(\mathcal{A}), A)=0$ for all $p \neq n$.

Proof. We use Theorem 2.1 and imitate the proof of [13, Theorem 6.3]. For each $X \in L(\mathcal{A})$, we recall that the restriction of the Hopf fibration $\mathbb{C}^{*} \rightarrow M\left(T \mathcal{A}_{X}\right) \rightarrow$ $U\left(T \mathcal{A}_{X}\right)$ identifies a central, cyclic subgroup in $G_{X}$; let $\gamma_{X} \in Z\left(G_{X}\right)$ be a generator. By the MCM hypothesis and the discussion from [13, Section 4.2], the coinvariant module $A_{\gamma_{X}}$ is an MCM module over $\pi_{1}\left(U\left(T \mathcal{A}_{X}\right)\right)=G_{X} /\left\langle\gamma_{X}\right\rangle$, 
and

$$
H^{i}\left(M\left(T \mathcal{A}_{X}\right), A\right) \cong H^{i-1}\left(U\left(T \mathcal{A}_{X}\right), A_{\gamma_{X}}\right)
$$

for all $i>0$. Furthermore, this latter group vanishes for $i \neq r(X)$, by [13, Theorem 5.6]. By hypothesis, though, $M\left(\mathcal{A}^{X}\right)$ is a Stein manifold of (complex) dimension $n-r(X)$. Thus, the factor of (4) indexed by $X$ is zero for $p+r(X)<$ $n-r(X)$.

Combining these facts, we see that $E_{2}^{p q}=0$, unless $p+q \geqslant n$; therefore, $H^{p+q}(M, A)=0$, unless $p+q \geqslant n$. On the other hand, $M=M(\mathcal{A})=M\left(\mathcal{A}_{\hat{0}}\right)$ is itself Stein, so $H^{p+q}(M, A)=0$ unless $p+q \leqslant n$. The conclusion follows.

REMARK 2.8. The Stein hypothesis in Theorem 2.7 is indispensable. Indeed, let $X=\mathbb{C}^{n}$, with $n \geqslant 2$, and let $\mathcal{A}=\{0\}$. Then the complement $U=\mathbb{C}^{n} \backslash\{0\}$ is not Stein, and also not an abelian duality space, since $U \simeq S^{2 n-1}$. Nevertheless, complements of hypersurfaces in Stein manifolds are again Stein [15, Satz 1].

2.5. Vanishing of twisted cohomology. As an application of Theorem 2.7, we can now prove the first theorem from the Introduction.

Proof of Theorem 1.1. Let $G=\pi_{1}(U)$. From the definition, we need to show that $H^{p}(U, A)=0$ for $p \neq n$, and $H^{n}(U, A)$ is torsion-free, for $A=\mathbb{Z}[G]$ and $A=\mathbb{Z}\left[G^{\mathrm{ab}}\right]$.

For this, we need to check that $A$ is an MCM module. By our injectivity result (Lemma 2.4), the restriction of $A$ to $\mathbb{Z}\left[C_{S, X}\right]$ is a free module, for each local free abelian group $C_{S, X}$, and free modules are MCM. Applying Theorem 2.7 completes the proof.

Another application is the following vanishing result, which proves Theorem 1.2 from the Introduction. Recall that, for each stratum $X \in L(\mathcal{A})$ one can choose a building set $\mathcal{G}_{X}$ : for each $g \in \mathcal{G}_{X}$ there is a corresponding boundary divisor in the wonderful model for $T \mathcal{A}_{X}$, as well as a generator for each free abelian group $C_{S}$ for which $g \in S$. Let $\gamma_{g} \in G$ denote the image of such a generator under the homomorphism $\alpha_{X, S}$ from Lemma 2.4.

COROLlARY 2.9. Let $\mathcal{A}$ be an arrangement of hypersurfaces satisfying the hypotheses of Theorem 2.7. Suppose $A$ is a finite-dimensional representation of $G=\pi_{1}(M(\mathcal{A}))$ over a field $\mathbb{k}$. If $A^{\gamma_{g}}=0$ for all $g \in \bigcup_{X \in L(\mathcal{A})} \mathcal{G}_{X}$, then $H^{i}(M(\mathcal{A}), A)=0$ for all $0 \leqslant i<n$.

Proof. First we note that the hypothesis makes sense: $\gamma_{g} \in G$ is only defined up to conjugacy; however, the property that 1 is not an eigenvalue of the representating 
matrix for $\gamma_{g}$ is invariant under conjugation. As noted in [13, Lemma 5.7], the hypothesis implies that the $\mathbb{k}[G]$-module $A$ is MCM, and Theorem 2.7 applies.

REMARK 2.10. The vanishing of cohomology in Corollary 2.9 is generic, in the following sense. For each $g$ in some building set $\mathcal{G}_{X}$, the representations $A \in \operatorname{Hom}\left(G, \mathrm{GL}_{r}(\mathbb{k})\right)$ for which $A^{g}=0$ form a Zariski open set. Since there are finitely many such $g$, the representations $Z \subseteq \operatorname{Hom}\left(G, \mathrm{GL}_{r}(\mathbb{k})\right)$ that satisfy the hypotheses of the corollary above form an open subvariety. If $Z$ is nonempty and the representation variety is irreducible, then $Z$ is Zariski-dense.

2.6. Vanishing of $\boldsymbol{L}^{2}$-cohomology. Finally, we show that the same hypotheses also imply Theorem 1.3 from the Introduction. We refer to the survey of Eckmann [17] and the book of Lück [34] for background on the subject. Related vanishing results can be found in $[9,10]$ (but see the caveat from the Introduction), as well as in the recent preprints $[32,33]$.

Once again, let $\mathcal{A}$ be an arrangement of hypersurfaces in a compact, complex, smooth variety $Y$ of dimension $n$, and let $G=\pi_{1}(M(\mathcal{A})$ ).

TheOREM 2.11. Suppose that $M\left(\mathcal{A}^{X}\right)$ is Stein for each $X \in L(\mathcal{A})$. Then the reduced $L^{2}$-cohomology groups ${ }^{\text {red }} H^{i}\left(M(\mathcal{A}), \ell_{2}(G)\right)$ vanish for all $i \neq n$.

Proof. We follow the approach of [7], which we explain briefly here. Let $\mathcal{N}(G)$ denote the group von Neumann algebra of $G$; that is, the algebra of (right) $G$ invariant bounded endomorphisms of $\ell_{2}(G)$. We have an algebra homomorphism $\mathbb{C}[G] \rightarrow \mathcal{N}(G)$ sending $g \in G$ to left multiplication by $g$. Instead of working as usual in the abelian category of $\mathbb{C}[G]$-modules, we let $\mathscr{T}$ be the subcategory of $\mathscr{N}(G)$-modules of dimension zero, and $\mathscr{E}$ be the (Serre) quotient of $\mathscr{N}(G)$ modules by $\mathscr{T}$. Then it is known that $\mathscr{E}$ is an abelian category and the quotient construction is exact, so our spectral sequence computation of $H^{\bullet}\left(M(\mathcal{A}), \ell_{2}(G)\right)$ may be carried out in $\mathscr{E}$.

To show that the reduced $L^{2}$-cohomology of the universal cover of $M(\mathcal{A})$ is concentrated in cohomology degree $n$, we compute in $\mathscr{E}$. For this, suppose $C_{S, X}$ is a free abelian subgroup generated by $\gamma_{1}, \ldots, \gamma_{r}$ as in Definition 2.6, where $r=|S|$. Let $R=\mathbb{C}\left[C_{S, X}\right]$. In order to imitate the proof of Theorem 2.7, we will show that

$$
{ }^{\mathrm{red}} H^{i}\left(C_{S, X}, \ell_{2}(G)\right)=0 \quad \text { in } \mathscr{E} \text { for all } i .
$$

For this, let $V=\left(\gamma_{1}-1\right) \ell_{2}(G)$, and we show that $\bar{V}=\ell_{2}(G)$, where ${ }^{-}$ denotes closure in the $\ell_{2}$-topology. Noting that $\ell_{2}(G)$ is a Hilbert space with an 
orthonormal basis $\{g: g \in G\}$, we can do this by checking that $\bar{V}^{\perp}=V^{\perp}=0$, as follows. For any $c \in \ell_{2}(G)$, we may write

$$
c=\sum_{i \in \mathbb{Z}, \mathbb{Z} g \in \mathbb{Z} \backslash G} c_{g, i} \gamma_{1}^{i} g,
$$

for some coefficients $c_{g, i} \in \mathbb{C}$, choosing right coset representatives for $\mathbb{Z} \cong\left\langle\gamma_{1}\right\rangle$. If $c \in V^{\perp}$, then

$$
\begin{aligned}
0 & =\left\langle c, \gamma_{1}^{i} g-\gamma_{1}^{i-1} g\right\rangle \quad \text { for all } i \in \mathbb{Z}, \text { and } \mathbb{Z} g \in \mathbb{Z} \backslash G, \\
& =c_{g, i}-c_{g, i-1} .
\end{aligned}
$$

So, for each $g$, the coefficient $c_{g, i}$ is independent of $i$. Since by assumption $\sum\left|c_{g, i}\right|^{2}<\infty$, it must be the case that $c_{g, i}=0$ for all $i$, which implies $c=0$.

Similarly, we show that, for $c \in \ell(G)$, if $\left(\gamma_{1}-1\right) c=0$, then $c=0$. Writing $c$ as in (11), we see then $\gamma_{1} c=c$ if and only if $c_{g, i}=c_{g, i+1}$ for all $i$. Once again, square summability implies each $c_{g, i}=0$.

Combining, we obtain a short exact sequence

$$
0 \longrightarrow \ell_{2}(G) \stackrel{\gamma_{1}-1}{\longrightarrow} \ell_{2}(G) \longrightarrow \ell_{2}(G) / V \longrightarrow 0,
$$

where $\ell_{2}(G) / V \in \mathscr{T}$. Applying $H^{\bullet}\left(C_{S, X},-\right)$ to (12) gives a long exact sequence, which reduces to isomorphisms

$$
0 \longrightarrow{ }^{\operatorname{red}} H^{i}\left(C_{S, X}, \ell_{2}(G)\right) \stackrel{H\left(\gamma_{1}-1\right)}{\longrightarrow}{ }^{\operatorname{red}} H^{i}\left(C_{S, X}, \ell_{2}(G)\right) \longrightarrow 0
$$

in the quotient category $\mathscr{E}$ for each $i$.

On the other hand, $\gamma_{1}-1$ is in the augmentation ideal of $C_{S, X}$, so it acts by zero on $H^{i}\left(C_{S, X}, A\right)$ for any coefficient module $A$, hence also on its image in $\mathscr{E}$. It follows that ${ }^{\text {red }} H^{i}\left(C_{S, X}, \ell_{2}(G)\right)=0$, for each $i$, as required.

\section{Applications}

3.1. Linear, toric, and elliptic arrangements. We need the following consequence of a result due to De Concini and Gaiffi [11].

Proposition 3.1. If $\mathcal{A}$ is a toric arrangement in $\mathbb{T}:=\left(\mathbb{C}^{*}\right)^{n}$, there exist a compactification $Y$ and an arrangement of subvarieties $\mathcal{L}$ in $Y$ for which $U(\mathcal{A})=Y \backslash \bigcup_{K \in \mathcal{L}} K$ and, for each connected stratum $X \in L(\mathcal{L})$, the relative interior $U\left(\mathcal{A}^{X}\right) \subseteq X$ is a Stein manifold. 
Proof. De Concini and Gaiffi [11] construct a compact toric variety $Y=Y_{\Delta}$ for which $U(\mathcal{A})=Y \backslash \bigcup_{K \in \mathcal{L}} K$ and $\mathcal{L}$ is an arrangement of subvarieties. Given a stratum $X \in L(\mathcal{L})$, we may write it as $X=\overline{g K} \cap Y_{C}$, where $g K \in L(\mathcal{A})$ is a coset of a torus in $\mathbb{T}$, and $Y_{C}$ is a closed torus orbit in $Y$, indexed by a (closed) cone $C$ in $\Delta$, the rational fan of $Y_{\Delta}$.

Let $N=\operatorname{Hom}\left(\mathbb{C}^{*}, \mathbb{T}\right)$ denote the lattice of 1-parameter subgroups, and let $V_{K}$ denote the subspace of $N \otimes_{\mathbb{Z}} \mathbb{R}$ given by restriction to the subtorus $K$. By [11, Theorem 3.1], the closure $\overline{g K}$ is again a toric variety, and $X$ is an affine toric subvariety corresponding to the chamber $C$ in $V_{K}$ [11, Proposition 3.1]. Then

$$
\begin{aligned}
U\left(\mathcal{A}^{X}\right) & =X \backslash\left(\bigcup_{\substack{F \in \mathcal{L}: \\
F \nsupseteq g K}} \bar{F} \cup \bigcup_{\substack{C^{\prime} \in \Delta: \\
C^{\prime} \nsubseteq C}} Y_{C^{\prime}}\right) \\
& =g K \backslash \bigcup_{\substack{F \in \mathcal{L}: \\
F \nsupseteq g K}} F,
\end{aligned}
$$

which is the complement of a toric arrangement in the torus $g K$. Since toric arrangement complements are affine varieties, we conclude that $U\left(\mathcal{A}^{X}\right)$ is a Stein manifold.

REMARK 3.2. The proof of Proposition 3.1 relies on a special recursive property of the construction from [11]. In general, though, complements of hypersurfaces in toric varieties need not be Stein (see, however, [40] for an interesting case in which they are).

REMARK 3.3. It should also be noted that Stein manifolds need not be abelian duality spaces. Indeed, let $X$ be the complement of a hypersurface in $\mathbb{C}^{n}$ with $k$ components, where $k<n, n \geqslant 2$, and $b_{n}(X)>0$. Then $X$ is Stein and has homological dimension $n$, but $b_{1}(X)=k$, and so $X$ cannot be an abelian duality space of dimension $n$, by [14, Proposition 5.9].

As a concrete example, let $X$ be the complement of the irreducible hypersurface $\mathbb{C}^{3}$ defined by the Brieskorn polynomial $f=x^{3}+y^{3}+z^{3}$. Then the Milnor fiber of $f$ is homotopic to a wedge of eight spheres, while the characteristic polynomial of the algebraic monodromy is $\Delta(t)=(t-1)^{2}\left(t^{2}+t+1\right)^{3}$. The Wang exact sequence of the Milnor fibration now shows that $b_{1}(X)=1$ and $b_{2}(X)=b_{3}(X)=2$.

We are now in a position to prove the second theorem from the Introduction.

Proof of Theorem 1.4. (1) If $M(\mathcal{A}) \subseteq \mathbb{C}^{n}$ is the complement of an affine-linear arrangement, by adding a hyperplane, it is also the complement of an arrangement 
in $\mathbb{P}^{n}$, and this result appeared in [13, Theorem 5.6]. We note that central arrangement complements are a special case.

(2) This result was also reported in [13, Corollary 6.4]; however, the proof given there is incomplete. One reduces to the essential case and notes that the restrictions $\mathcal{A}^{X}$ are again essential, hence Stein, by [13, Proposition 6.1]. To verify that $\mathbb{Z}[G]$ and $\mathbb{Z}\left[G^{\text {ab }}\right]$ are MCM, one needs to know that the maps $\alpha_{X, S}: C_{X, S} \rightarrow$ $G$ are injective, the proof of which was omitted, but is now covered by Lemma 2.4.

(3) By Proposition 3.1, the toric arrangement complement admits a compactification, which satisfies the conditions of Theorem 1.1. The claim follows.

3.2. (Orbit) configuration spaces of Riemann surfaces. Let $\Gamma$ be a discrete group that acts freely and properly discontinuously on a space $X$. The orbit configuration space $F_{\Gamma}(X, n)$ is, by definition, the subspace of the cartesian product $X^{\times n}$ consisting of $n$-tuples $\left(x_{1}, \ldots, x_{n}\right)$ for which the $\Gamma$-orbits of $x_{i}$ and $x_{j}$ are disjoint for all $1 \leqslant i \neq j \leqslant n$. If $|\Gamma|=1$, then $F_{\Gamma}(X, n)=F(X, n)$, the classical (ordered) configuration space. Orbit configuration spaces were first investigated in the thesis of Xicoténcatl [39], and further studied, for instance, in $[5,6]$.

In the case when $X=M$ is a smooth manifold of dimension $d$, and $\Gamma$ acts by diffeomorphisms, the orbit configuration space $F_{\Gamma}(M, n)$ is a smooth manifold of dimension $d n$. Perhaps the most studied case (and the one we are mainly interested here) is when $M=\Sigma_{g, k}$ is a Riemann surface of genus $g$ with $k \geqslant 0$ punctures, and $\Gamma$ is finite. Note that, if $k=0$, the complement in $\Sigma_{g}^{\times n}$ of $F_{\Gamma}\left(\Sigma_{g}, n\right)$ is the union of an arrangement of smooth, complex algebraic hypersurfaces.

Xicoténcatl showed that the classical Fadell and Neuwirth [20] fibration applies in the more general case of orbit configuration spaces:

$$
F_{\Gamma}\left(\Sigma_{g, k+|\Gamma|}, n-1\right) \longrightarrow F_{\Gamma}\left(\Sigma_{g, k}, n\right) \longrightarrow \Sigma_{g, k} .
$$

Consider the 'tautological' compactification of the orbit configuration space $U=F_{\Gamma}\left(\Sigma_{g, k}, n\right)$, namely $Y=\Sigma_{g}^{\times n}$. The components of the boundary divisor, $D=Y \backslash U$, form an arrangement of hypersurfaces,

$$
\mathcal{B}_{n}:=\left\{H_{i j}^{\gamma} \mid \gamma \in \Gamma, 1 \leqslant i \neq j \leqslant n\right\} \cup\left\{K_{i, l} \mid 1 \leqslant i \leqslant n, 1 \leqslant l \leqslant k\right\},
$$

where $H_{i j}^{\gamma}$ is given by the equation $x_{i}=\gamma \cdot x_{j}$ and $K_{i, l}$ by $x_{i}=p_{l}$, where $p_{1}, \ldots$, $p_{k} \in \Sigma_{g}$ are the punctures of $\Sigma_{g, k}$.

The intersection poset $L\left(\mathcal{B}_{n}\right)$ can be described in terms of labeled partitions via a slight generalization of the Dowling lattice. To describe it, we write $\Pi \vDash[n]$ to 
denote a set partition $\Pi$ of $[n]$. We regard $\Pi$ as a category whose objects are the set $[n]$, and for which there is a (unique) morphism $i \rightarrow j$ if and only if $i \sim j$ in $\Pi$. The action of $\Gamma$ on $\Sigma_{g, k}$ extends continuously to an action on $\Sigma_{g}$. We let $C:=C\left(\Gamma, \Sigma_{g}\right)$ denote the category whose objects are $\left\{\Sigma_{g, k},\left\{p_{1}\right\}, \ldots,\left\{p_{k}\right\}\right\}$, a $\Gamma$ equivariant partition of $\Sigma_{g}$. For a pair of objects $S, T$, by definition $[\gamma]: S \rightarrow T$ is a morphism whenever $[\gamma] \in \Gamma / \operatorname{stab}(S)$ and $\gamma(S) \subseteq T$. Composition is induced by the group operation.

Given a point $x \in Y=\Sigma_{g}^{\times n}$, let $\Pi_{x}$ be the partition determined by $x$ for which $i \sim j$ if and only if $\left(\Gamma \cdot x_{i}\right) \cap\left(\Gamma \cdot x_{j}\right)$ is nonempty. Then $x_{i}=\gamma_{i j} x_{j}$ for some $\gamma_{i j} \in \Gamma$. For a point $x \in \Sigma_{g}$, we let $[x]$ be the object of $C$ containing $x$. Define a functor $f_{x}: \Pi_{x} \rightarrow C$ by $f(i)=\left[x_{i}\right]$, for each $i \in[n]$, and let $f_{x}(j \rightarrow i)=\left[\gamma_{i j}\right]$, for all $i \sim j$. It is easy to check that $f_{x}$ is well defined, and has the following property.

Lemma 3.4. For all $i, j \in[n]$, a point $x \in Y$ is on the hypersurface $H_{i j}^{\gamma}$ if and only if $f_{x}(j \rightarrow i)=\left[\gamma_{i j}\right]$. A point $x \in Y$ is on the hypersurface $K_{i, l}$ if and only if $f_{x}(i)=\left\{p_{l}\right\}$.

Now we can describe the stratification of $Y$. Let

$$
\mathcal{P}_{n}=\{(\Pi, f): \Pi \vDash[n], f \in \operatorname{Funct}(\Pi, C)\} .
$$

Consider the function $\Phi: Y \rightarrow \mathcal{P}_{n}$ given by $\Phi(x)=\left(\Pi_{x}, f_{x}\right)$. Let $X_{\Pi, f}=$ $\Phi^{-1}(\Pi, f)$ for each pair $(\Pi, f) \in \mathcal{P}_{n}$.

Proposition 3.5. For any pair $(\Pi, f) \in \operatorname{im}(\Phi)$, the space $X_{\Pi, f}$ coincides with $M\left(\mathcal{B}_{n}^{X}\right)$, where

$$
\begin{aligned}
X & =\overline{X_{\Pi, f}} \\
& =\bigcap_{\substack{i, j, \gamma: \\
f(j \rightarrow i)=\left[\gamma_{i j}\right]}} H_{i j}^{\gamma} \cap \bigcap_{\substack{i, l: \\
f(i)=\left\{p_{l}\right\}}} K_{i, l} .
\end{aligned}
$$

Proof. By Lemma 3.4, a point $x$ belongs to $X_{\Pi, f}$ if and only if it is in the intersection (17), and $x$ is not contained in any other hypersurface. But this is exactly the space $M\left(\mathcal{B}_{n}^{X}\right)$.

Proposition 3.6. If $k>0$, then for each $X \in L\left(\mathcal{B}_{n}\right)$, the complement $M\left(\mathcal{B}_{n}^{X}\right)$ is a Stein manifold.

Proof. First we check the case $X=Y$, where $\mathcal{B}_{n}^{X}=\mathcal{B}_{n}$. Here, $M\left(\mathcal{B}_{n}\right)$ is a hypersurface complement in $\Sigma_{g, k}^{\times n}$. As mentioned previously, the product of open 
Riemann surfaces is Stein, and a hypersurface complement in a Stein manifold is again Stein; thus, the base case is proved.

In general, for a stratum $X_{\Pi, f}$ defined above, it is easy to see from Proposition 3.5 that the arrangement $\mathcal{B}_{n}^{X}$ is again an orbit configuration space, $F_{\Gamma}\left(\Sigma_{g, k}, m\right)$, where $m$ is the number of diagonal blocks of $\Pi$. This reduces the claim to the case $X=Y$ treated above.

Now we may characterize the duality properties of orbit configuration spaces of points in Riemann surfaces.

Proof of Theorem 1.5. For $g>0$ and $k>0$, we see that $F_{\Gamma}\left(\Sigma_{g, k}, n\right)$ is both a duality space and an abelian duality space of dimension $n$ : by Proposition 3.6, the configuration space satisfies the hypotheses of Theorem 1.1.

For $k=0$, we note that $\Sigma_{g}$ is a (Poincaré) duality space of dimension 2 . Noting also that $F_{\Gamma}\left(\Sigma_{g, 1}, 1\right)=\Sigma_{g, 1}$, we see that $F_{\Gamma}\left(\Sigma_{g}, n\right)$ is a duality space of dimension $n+1$ for all $n \geqslant 1$ by induction, using the fibration sequence (14) and a classical result of Bieri and Eckmann [3, Theorem 3.5].

If $k=0$ and $g=1$, the configuration space $F_{\Gamma}\left(\Sigma_{g}, n\right)$ is an elliptic arrangement complement, hence an abelian duality space by Theorem 1.4. However, if $k=0$ and $g \geqslant 2$, it need not be an abelian duality space. The (easy) case $n=1$ appears as [14, Example 5.8]: $\Sigma_{g, k}$ is an abelian duality space if and only if $k \geqslant 1$. For $n \geqslant 2$, we restrict our attention to the case where $\Gamma$ is trivial (and $k=0$ ).

Again from the fibration sequence (14) and by induction on $n$ we see that $F\left(\Sigma_{g}, n\right)$ is a CW-complex of dimension at most $n+1$. Furthermore, it is known that $b_{3}\left(F\left(\Sigma_{g}, 2\right)\right)=2 g$ (see e.g. [1, Corollary 12]) and that $b_{n+1}\left(F\left(\Sigma_{g}, n\right)\right) \neq 0$, for all $n \geqslant 3$ (see [35, Proposition 1.4]). Thus, if $F\left(\Sigma_{g}, n\right)$ were to be an abelian duality space, it would have to be of (formal) dimension $n+1$. On the other hand, by [25], the generating series for the Euler characteristics of the unordered configuration spaces of $\Sigma_{g}$ is given by

$$
1+\sum_{n \geqslant 1} \chi\left(C\left(\Sigma_{g}, n\right)\right) t^{n}=(1+t)^{2-2 g} .
$$

Therefore, $(-1)^{n} \chi\left(C\left(\Sigma_{g}, n\right)\right)>0$, and hence $(-1)^{n} \chi\left(F\left(\Sigma_{g}, n\right)\right)>0$, also. In view of (2), we conclude that $F\left(\Sigma_{g}, n\right)$ is not an abelian duality space.

Last, we consider the genus $g=0$ case. If $k>0$, then $F_{\Gamma}\left(\Sigma_{0, k}, n\right)$ is an affinelinear hyperplane arrangement complement of corank 0. By Theorem 1.4 again, it is both a duality space and an abelian duality space of dimension $n$.

It remains to show that if $g=0$ and $k=0$, then $F\left(\Sigma_{0}, n\right)$ is neither a duality nor an abelian duality space. For $n \leqslant 2$, this is clear, since then $F\left(\Sigma_{0}, n\right) \simeq S^{2}$ from (14). For $n \geqslant 3$, we have (see, e.g., [23])

$$
F\left(\Sigma_{0}, n\right) \cong \mathrm{PGL}_{2}(\mathbb{C}) \times F\left(\Sigma_{0,2}, n-2\right),
$$


where $F\left(\Sigma_{0,2}, n-2\right)$ is the complement of an affine-linear hyperplane arrangement in $\mathbb{C}^{n-2} \subseteq \mathbb{P}^{n-2}$. This is both a duality space and an abelian duality space but $\mathrm{PGL}_{2}(\mathbb{C}) \simeq \mathrm{SO}(3)$ is neither, from which the conclusion follows.

\section{Acknowledgements}

We would like to thank Giovanni Gaiffi for helping us apply his work with Corrado De Concini to our Proposition 3.1, and Stefan Papadima for help with Lemma 2.3. We also thank the referees for their careful reading of our manuscript and for their many insightful comments and suggestions. G.D. was supported by NSERC of Canada. A.I.S. was partially supported by Simons Foundation collaborative grant 354156 . We gratefully acknowledge support by the Research in Pairs program during our stay at the Mathematisches Forschungsinstitut Oberwolfach in May-June 2016. G.D. would also like to thank the University of Sydney School of Mathematics and Statistics for its hospitality.

\section{References}

[1] H. Azam, 'Cohomology groups of configuration spaces of Riemann surfaces', Bull. Math. Soc. Sci. Math. Roumanie (N.S.) 58(1) (2015), 33-47.

[2] C. Bibby, 'Cohomology of abelian arrangements', Proc. Amer. Math. Soc. 144(7) (2016), 3093-3104.

[3] R. Bieri and B. Eckmann, 'Groups with homological duality generalizing Poincaré duality', Invent. Math. 20 (1973), 103-124.

[4] N. Budur, 'Complements and higher resonance varieties of hyperplane arrangements', Math. Res. Lett. 18(5) (2011), 859-873.

[5] K. Casto, ' $\mathrm{FI}_{g}$-modules, orbit configuration spaces, and complex reflection groups', Preprint, 2016, arXiv:1608.06317v1.

[6] F. R. Cohen, T. Kohno and M. A. Xicoténcatl, 'Orbit configuration spaces associated to discrete subgroups of PSL(2, $\mathbb{R})$ ', J. Pure Appl. Algebra 213(12) (2009), 2289-2300.

[7] M. W. Davis, T. Januszkiewicz and I. J. Leary, 'The $l^{2}$-cohomology of hyperplane complements', Groups Geom. Dyn. 1(3) (2007), 301-309.

[8] M. W. Davis, 'Complements of hyperplane arrangements as posets of spaces', Preprint, 2015, arXiv:1502.03650v2, comment and abstract.

[9] M. W. Davis, T. Januszkiewicz, I. J. Leary and B. Okun, 'Cohomology of hyperplane complements with group ring coefficients', Int. Math. Res. Not. IMRN 9 (2011), 2110-2116.

[10] M. W. Davis and S. Settepanella, 'Vanishing results for the cohomology of complex toric hyperplane complements', Publ. Mat. 57(2) (2013), 379-392.

[11] C. De Concini and G. Gaiffi, 'Projective wonderful models for toric arrangements', Preprint, 2016, arXiv:1608.08746v1.

[12] C. De Concini and C. Procesi, 'Wonderful models of subspace arrangements', Selecta Math. (N.S.) 1(3) (1995), 459-494. 
[13] G. Denham, A. I. Suciu and S. Yuzvinsky, 'Combinatorial covers and vanishing of cohomology', Selecta Math. (N.S.) 22(2) (2016), 561-594.

[14] G. Denham, A. I. Suciu and S. Yuzvinsky, 'Abelian duality and propagation of resonance', Selecta Math. (N.S.) 23(4) (2017), 2331-2367.

[15] F. Docquier and H. Grauert, 'Levisches Problem und Rungescher Satz für Teilgebiete Steinscher Mannigfaltigkeiten', Math. Ann. 140 (1960), 94-123.

[16] C. Dupont, 'The Orlik-Solomon model for hypersurface arrangements', Ann. Inst. Fourier (Grenoble) 65(6) (2015), 2507-2545.

[17] B. Eckmann, 'Introduction to $\ell_{2}$-methods in topology: reduced $\ell_{2}$-homology, harmonic chains, $\ell_{2}$-Betti numbers', Israel J. Math. 117(1) (2000), 183-219.

[18] H. Esnault, V. Schechtman and E. Viehweg, 'Cohomology of local systems on the complement of hyperplanes', Invent. Math. 109(3) (1992), 557-561.

[19] A. Esterov and K. Takeuchi, 'On vanishing theorems for local systems associated to Laurent polynomials', Nagoya J. Math. (2017), 1-22. doi:10.1017/nmj.2017.8.

[20] E. Fadell and L. Neuwirth, 'Configuration spaces', Math. Scand. 10 (1962), 111-118.

[21] E. M. Feichtner, 'De Concini-Procesi wonderful arrangement models: a discrete geometer's point of view', in Combinatorial and Computational Geometry, Mathematical Sciences Research Institute Publications, 52 (Cambridge University Press, Cambridge, 2005), 333-360.

[22] E. M. Feichtner and S. Yuzvinsky, 'Chow rings of toric varieties defined by atomic lattices', Invent. Math. 155(3) (2004), 515-536.

[23] E. M. Feichtner and G. M. Ziegler, 'The integral cohomology algebras of ordered configuration spaces of spheres', Doc. Math. 5 (2000), 115-139.

[24] Y. Félix, S. Halperin and J.-C. Thomas, Rational Homotopy Theory, Graduate Texts in Mathematics, 205 (Springer, New York, 2001).

[25] Y. Félix and J.-C. Thomas, 'Rational Betti numbers of configuration spaces', Topology Appl. 102(2) (2000), 139-149.

[26] F. Forstnerič, Stein Manifolds and Holomorphic Mappings. The Homotopy Principle in Complex Analysis, Ergebnisse der Mathematik und ihrer Grenzgebiete, 56 (Springer, Heidelberg, 2011).

[27] K. Fritzsche and H. Grauert, From Holomorphic Functions to Complex Manifolds, Graduate Texts in Mathematics, 213 (Springer, New York, 2002).

[28] P. Griffiths and J. Morgan, Rational Homotopy Theory and Differential Forms, second edition, Progress in Mathematics, 16 (Springer, New York, 2013).

[29] T. Kohno, 'Homology of a local system on the complement of hyperplanes', Proc. Japan Acad. Ser. A Math. Sci. 62(4) (1986), 144-147.

[30] A. Levin and A. Varchenko, Cohomology of the Complement to an Elliptic Arrangement, Configuration spaces, CRM Series, 14 (2012), 373-388. Ed. Norm., Pisa.

[31] L. Li, 'Wonderful compactification of an arrangement of subvarieties', Michigan Math. J. 58(2) (2009), 535-563.

[32] Y. Liu, L. Maxim and B. Wang, 'Topology of subvarieties of complex semi-abelian varieties', Preprint, 2017, arXiv:1706.07491v1.

[33] Y. Liu, L. Maxim and B. Wang, 'Mellin transformation, propagation, and abelian duality spaces', Preprint, 2017, arXiv:1709.02870v2.

[34] W. Lück, $L^{2}$-invariants: Theory and Applications to Geometry and K-theory, Ergebnisse der Mathematik und ihrer Grenzgebiete. (3), 44 (Springer, Berlin, 2002).

[35] M. Maguire, 'Computing cohomology of configuration spaces (with an appendix by Matthew Christie and Derek Francour)', Preprint, 2016, arXiv:1612.06314v1. 
[36] V. Schechtman, H. Terao and A. Varchenko, 'Local systems over complements of hyperplanes and the Kac-Kazhdan conditions for singular vectors', J. Pure Appl. Algebra 100(1-3) (1995), 93-102.

[37] A. I. Suciu and H. Wang, 'Formality properties of finitely generated groups and Lie algebras', Preprint, 2015, arXiv:1504.08294.

[38] B. Totaro, 'Configuration spaces of algebraic varieties', Topology 35(4) (1996), 1057-1067.

[39] M. A. Xicoténcatl Merino, 'Orbit configuration spaces, infinitesimal braid relations in homology and equivariant loop spaces', PhD Thesis, University of Rochester, 1997.

[40] O. V. Yakovleva, 'On the Stein property of the complement of an algebraic surface in a toric manifold', Sibirsk. Mat. Zh. 39(3) (1998), 703-713. 